\title{
Dietary fibre intake and risk of fatal coronary heart disease in a cohort of British women
}

\author{
D. E. Threapleton ${ }^{1}$, V. J. Burley ${ }^{1}$, D. C. Greenwood ${ }^{2}$, M. Aldwairji ${ }^{1}$ and J. E. Cade ${ }^{1}$ \\ ${ }^{1}$ School of Food Science \& Nutrition, University of Leeds, Leeds LS2 9JT and ${ }^{2}$ Centre for Epidemiology \& Biostatistics, \\ University of Leeds, Leeds, LS2 9JT, UK
}

Coronary heart disease (CHD) remains a significant problem and one of the main causes of death within the United Kingdom today, accounting for over 88,000 deaths per year ${ }^{(1)}$. Dietary fibre is thought to influence CHD risk through changes in key modifiable risk factors such as body weight, blood cholesterol, or blood pressure ${ }^{(2)}$. Exploring intakes of different foods sources of fibre could prove beneficial for tailoring public health messages to focus on more protective types of foods.

The UK Women's Cohort Study recruited more than 35,000 British women in 1995 and they have been followed for over 14 years on average. Diet for the previous 12 months was assessed at baseline using a 217-item food frequency questionnaire (FFQ). Risk of fatal CHD was explored in relation to total fibre intake estimated as non-starch polysaccharide (NSP) or using the Association Official Analytical Chemist method (AOAC) and also water-soluble and insoluble fibre. In addition, NSP from specific foods was explored in relation to CHD risk (cereal foods, fruit, vegetables, legumes and nuts/seeds). Cox regression survival analysis was used to estimate risk with increasing fibre intakes.

One hundred and thirteen incident CHD cases were included in models which were fully-adjusted for confounders (adjustments included age, body mass index (BMI), alcohol intake, physical activity, energy intake, smoking status and socio-economic status). None of the exposures were significantly associated with fatal CHD risk. Relative risk (RR) was 0.96 (95\% confidence intervals (CI) 0.79 to 1.17 , $p=0.69$ ) for every $6 \mathrm{~g} /$ day increase in NSP intake and was virtually the same for each $11 \mathrm{~g} /$ day increase of AOAC fibre 0.96 (95\% CI 0.73 to $1.26, p=0.76$ ). When the sample was divided into quintiles based on fibre intake, there appeared to be no pattern in risk estimates with greater fibre intake and CIs remained very wide (see table).

\begin{tabular}{llllll}
\hline $\begin{array}{l}\text { Mean NSP fibre } \\
\text { intake g/day }\end{array}$ & Cases & $\begin{array}{c}\text { RR }(95 \% \text { CI }) \\
\text { compared to Q1 }\end{array}$ & $p$-value & $\begin{array}{c}\text { Mean AOAC } \\
\text { fibre intake g/day }\end{array}$ & $\begin{array}{c}\text { RR (95\% CI) } \\
\text { compared to Q1 }\end{array}$ \\
\hline Q1: 14.1 & 24 & 1 & & Q1: 21.0 & 1 \\
Q2: 19.4 & 28 & $1.30(0.76,2.24)$ & 0.34 & Q2: 30.0 & 27 \\
Q3: 23.8 & 18 & $0.93(0.48,1.82)$ & 0.79 & Q3: 36.8 & 26 \\
Q4: 29.1 & 26 & $1.15(0.61,2.17)$ & 0.66 & Q4: 44.8 & $1.18(0.68,2.03)$ \\
Q5: 38.3 & 17 & $0.89(0.38,2.08)$ & 0.79 & Q5: 63.0 & 0.56 \\
\hline
\end{tabular}

Analyses stratified by baseline BMI (less than 25 or greater than $25 \mathrm{~kg} / \mathrm{m}^{2}$ ) or menopausal status did not provide any different results, with no exposures being associated with CHD risk. Analysis of CHD risk in relation to fibre sources did not generate any evidence of a clear pattern of association.

Results indicate that greater fibre intake did not influence CHD risk in this sample. However, the number of fatal CHD cases was relatively small and exploration of CHD incidence, in planned future research, may increase statistical power to detect an association or will confirm this result. This sample of women tended to be healthy with an average fibre intake greater than the national average. These results suggest that increasing fibre intake in these women failed to confer any additional protective benefit in terms of CHD fatality risk in those with healthy dietary habits.

1. Scarborough P, Bhatnagar P, Wickramasinghe K, Smolina K, Mitchell C, Rayner M (2010) Coronary heart disease statistics. British Heart Foundation: London.

2. Unal B, Critchley JA, Capewell S (2004) Explaining the decline in coronary heart disease mortality in England and Wales between 1981 and 2000. Circulation 109(9), 1101-1107. 